\title{
Distinct mechanisms for the representation of moving and static objects
}

\author{
Zoe Kourtzi \\ Department of Brain \& Cognitive Science, MIT, Cambridge, MA, USA and \\ Max-Planck Institute for Biological Cybernetics, Tübingen, Germany \\ Ken Nakayama \\ Vision Science Lab, Harvard University, Cambridge, MA, USA
}

\begin{abstract}
The visual system has been suggested to integrate different views of an object in motion. We investigated differences in the way moving and static objects are represented by testing for priming effects to previously seen ("known") and novel object views. We showed priming effects for moving objects across image changes (e.g., mirror reversals, changes in size, and changes in polarity) but not over temporal delays. The opposite pattern of results was observed for objects presented statically; that is, static objects were primed over temporal delays but not across image changes. These results suggest that representations for moving objects are: (1) updated continuously across image changes, whereas static object representations generalize only across similar images, and (2) more short-lived than static object representations. These results suggest two distinct representational mechanisms: a static object mechanism rather spatially refined and permanent, possibly suited for visual recognition, and a motion-based object mechanism more temporary and less spatially refined, possibly suited for visual guidance of motor actions.
\end{abstract}

Interacting in our natural environments entails perceiving objects moving continuously either because we move or the objects in our environment move. As a result, the images of objects that fall on our retinas vary over time in properties, such as the object's position, orientation, or size. Recognition of objects across these image changes has been extensively studied when the objects are presented statically (Biederman \& Gerhardstein, 1993; Cooper, Biederman, \&

Please address all correspondence to Z. Kourtzi, Max Planck Institute for Biological Cybernetics, Spemannstrass e 38, 72076 Tuebingen, Germany.Email: zoe.kourtzi@ tuebingen.mpg.d e

We would like to thank Shimon Ullman, Steven Yantis, and Tim McNamara for useful comments and discussions. We also thank Anne Grossetete for research assistance with the experiments. 
Hummel, 1992; Cooper \& Schacter, 1992; Cooper, Schacter, Ballesteros, \& Moore, 1992). But how does the visual system represent moving objects? That is, how are different images of a moving object integrated over time so we can perceive the same object moving continuously?

Several recent studies have addressed these questions by testing how information about motion and form is integrated in object representations. For example, the representational momentum effect, discussed in depth in this special issue, demonstrates that object displacement is represented as a continuous event in memory (Freyd, 1987; Freyd \& Johnson, 1987). Moreover, these dynamic object representations can be influenced by physical shape properties as well as conceptual information about the object (e.g., Freyd \& Pantzer, 1995; Reed \& Vinson, 1996; Vinson \& Reed, this issue). Similarly, rotational motion seems to be represented in terms of an object's structural axes (Shepard, 1981, 1984, 1988; Shepard \& Cooper, 1982; Shiffrar \& Shepard, 1991) and the structure of human form may influence interpretations of apparent motion (Shiffrar \& Freyd, 1990, 1993).

Recent human imaging studies have shown that motion sensitive areas (MT+/V5) are activated by static images of objects when these images imply motion of the depicted objects (Kourtzi \& Kanwisher, 2000a; Senior et al., 2000; Senior, Ward, \& David, this issue). Finally, accumulating evidence on the relationship between the motor and the visual system further supports the interdependence of motion and form information in object representations. In particular, the visual structure of an object may influence our motor interaction with that object; for example, the size, shape, and orientation of an object affects how it is grasped (Goodale \& Milner, 1992). Evidence supporting this proposal comes from neurophysiological (e.g., Sakata, Taira, Kusunoki, Murata, \& Tanaka, 1997; Sakata et al., 1998), imaging (e.g., Martin, Wiggs, Ungerleider, \& Haxby, 1996; Stevens, Fonlupt, Shiffrar, \& Decety, 2000) psychophysical (Goodale \& Milner, 1992) and patient studies (Goodale, Milner, Jackobson, \& Carey, 1991). Taken together, all these studies suggest that object representations are dynamic rather than static; that is, object representations may contain both motion and shape information in a linked fashion.

Consistent with this idea, previous studies (Kourtzi \& Shiffrar, 1997, 1999a, b, 2001) asked whether moving objects are represented in a different manner than static objects. Specifically, these studies used a priming paradigm, in which two rotated views of a prime object were presented statically or linked by apparent motion and they were followed by a pair of targets. The observers were asked to report whether the targets matched each other. Priming was indicated by faster reaction times when the two targets were the same as the prime object. A priming effect was observed when the tested views were the same as the views of the prime object ("known" views). For statically presented objects this effect generalized to novel views of the prime object. This occurred only for small rotation angles from the prime views and constituted a static 
generalization effect. However, when the same prime views were linked by apparent motion, priming was observed for novel views falling within (interpolation effect) but not outside (suppression effect) the object's motion path even for large rotation angles from the "known" prime views. These results suggest that the visual system may take advantage of the object's motion and link different views of an object even across large rotation changes. As a result, object views falling within the object's motion path are readily represented.

More importantly, these studies suggest that moving objects are represented in a different manner than static objects and that different mechanisms may underlie the representation of moving and static objects. In particular, the characteristic signature of a static object representational mechanism is the restricted generalization around "known" views. This mechanism has been suggested to be important for long-term recognition of objects as well as for discrimination of objects from similar exemplars of the same category (Bülthoff \& Edelman, 1992; Logothetis, Pauls, Bülthoff, \& Poggio, 1994; Logothetis, Pauls, \& Poggio, 1995; Poggio \& Edelman, 1990). However, the characteristic signature of a motion-based object representational mechanism is the interpolation of information within the object's motion path and the suppression of information outside this path. We propose that this mechanism may be important for the continuous updating of information about moving objects and for discriminating between objects moving along different paths. As a result, we know "what" object moved 'where' at a specific moment in time and we are able to guide our actions successfully towards the objects in the world.

\section{SIGNATURES OF THE REPRESENTATIONAL MECHANISMS FOR STATIC AND MOVING OBJECTS}

To convey the characteristic signatures of the static and motion-based object representational mechanisms we provide in Figure 1 a summary of the results from our previous studies (Kourtzi \& Shiffrar, 1997). We show priming data (indicated by the black dots) in polar coordinates for object views differing by a defined angle - small $\left(<90^{\circ}\right)$ and large $\left(>90^{\circ}\right)$ rotation angles - that were presented either as static images (static condition) or linked by apparent motion (motion condition). The polar representation of these data is particularly useful in showing that the generalization gradients associated with static views differ from gradients associated with views linked by motion.

Let's consider first the case of static objects depicted Figure 1a and b. The two angular views presented as primes are labelled frame 1 and frame 2 . Five angular views were presented as test. The test views consisted of the two prime views and three novel views; one novel view midway between the two prime views (inter) and two novel views (extra 1 and extra 2) outside the prime views. All the novel views were spaced equally from the prime views. The grey 


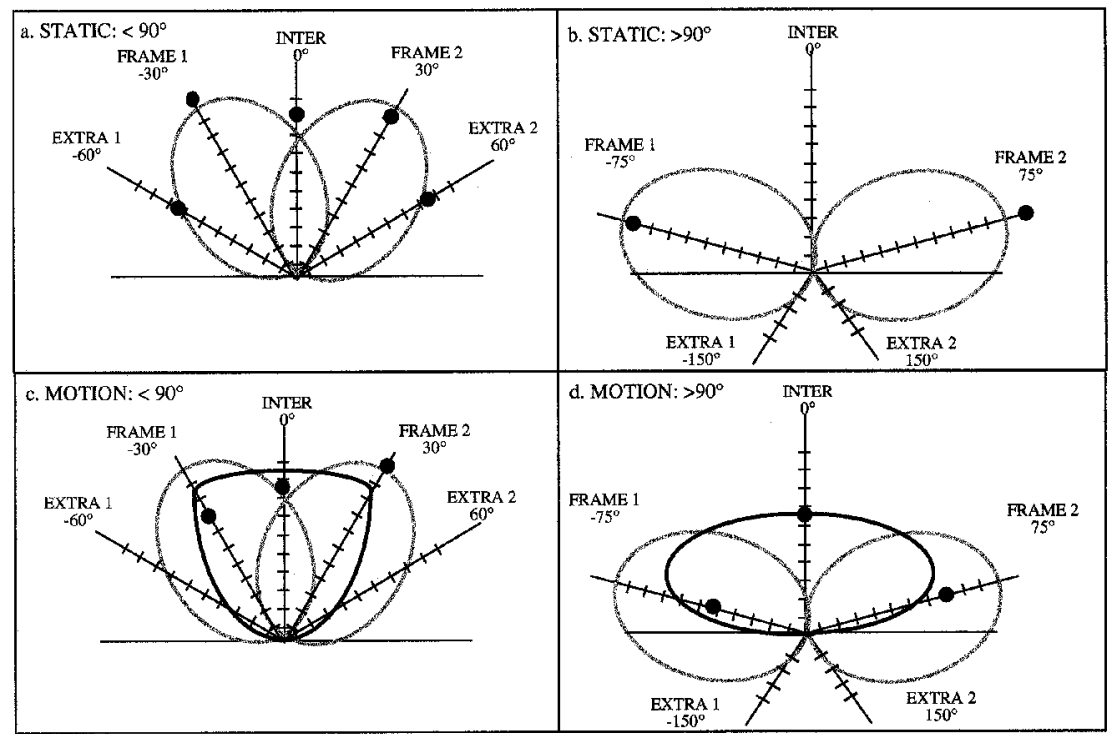

Figure 1. Theoretical summary of previous data (Kourtzi \& Shiffrar, 1997). Illustrations describing our predictions on the priming patterns based on the results of previous studies (Kourtzi \& Shiffrar, 1997). These radial plots illustrate amount of priming for each target orientation. Magnitude of priming is plotted in msec starting with $0 \mathrm{~ms}$ at the origin of the axes and increasing towards the periphery of the radial plots by steps of $10 \mathrm{~ms}$. That is, points close to the origin of the axes illustrate lack of priming, whereas points towards the periphery of the plots illustrate stronger priming. The black dots represent the amount of priming observed for "known" and novel views when objects were presented statically or in motion at small rotations (average of priming for $30^{\circ}, 60^{\circ}, 90^{\circ}$ ) and large rotations (average of priming for $120^{\circ}, 150^{\circ}$ ). Only significant effects are plotted, i.e., when the amount of priming was not significant for an orientation it is not plotted. The elliptical plots (grey lines) illustrate the generalization fields around "known" views, (frame 1 and frame 2) for static objects at rotation angles of (a) $60^{\circ}$ and (b) $150^{\circ}$ based on our previous data (Kourtzi \& Shiffrar, 1997) and as described in previous psychophysica 1 studies (e.g., Bülthoff \& Edelman, 1992). The solid black curve lines illustrate the effects observed in our previous studies for moving objects, i.e., interpolation in between the "known" views and suppression outside the "known" views in the motion condition for rotation angles of (c) $60^{\circ}$ and (d) $150^{\circ}$.

ellipses straddling the two prime views (frame 1 and frame 2) are best-fitting ellipses of the data from Kourtzi and Shiffrar (1997) and represent a summary estimate of the static generalization observed in this data. Of importance is that the generalization curves are sufficiently broad so that when the primes are separated by less than $90^{\circ}$ (Figure 1a) there is significant priming for the novel view between the prime views. This effect can be expected from the two static generalization curves for the "known" prime views. Similarly, there should also be significant priming for the frames outside the "known" prime views, as shown in this same panel. Note, however, that when the prime views are more than $90^{\circ}$ apart (Figure $1 \mathrm{~b}$ ), the novel views between as well as outside the "known" prime views fall outside the static generalization curve. Thus, Figure 
1a and $\mathrm{b}$ illustrates conditions where we expect priming effects due to static generalization (Figure 1a) and conditions where the static generalization fails and priming effects are not expected (Figure 1b). These effects constitute the characteristic signature of a static object representational system that is based on a restricted generalization across "known" views.

This pattern of results for static objects provides the background for understanding the effects observed when the prime stimuli activate a motion-based object representational system. Thus, now we can consider the case where the same prime views were linked by motion. These results are presented in Figure $1 \mathrm{c}$ and $\mathrm{d}$. Here the results are altogether different and cannot be predicted from the static generalization curves. Motion has two effects on the priming of novel object views. The first one is expressed as facilitation, such that there is priming for novel views in between the two "known" prime views (inter) more than $90^{\circ}$ apart suggesting a motion-based interpolation even for large rotation angles (Figure 1d). The second one is expressed as suppression, such that there is little or no priming outside of the motion trajectory (extra 1, extra 2) even for small rotation angles. Thus, we observe two consequences of apparent motion that are not predicted from the static generalization curves. That is, we observe interpolation within the object's motion path for large rotation angles between the "known" views and suppression outside the object's motion path for small rotation angles. These effects constitute the characteristic signature of a putative motion-based representational system where views within a trajectory seem to be potentiated and views outside show suppression.

\section{CURRENT EXPERIMENTS}

The goal of our current experiments was to delineate more clearly the distinction between mechanisms for the representation of moving and static objects. To this end, in two experiments we tested whether the signature of the static object representational mechanism (i.e., restricted generalization) and that of the motion-based object representational mechanism (i.e., the interpolation and suppression effects within and outside the object's motion path, respectively) differ under a range of conditions. In particular, we tested whether moving and static objects show different priming effects (1) across image changes, namely mirror reversal, size change, polarity change (Experiment 1) and (2) over temporal delays (Experiment 2). These experiments were motivated by the hypothesis that the static object system mediates long-term object recognition processes, whereas the motion-based system continuously updates information about objects for visual guidance of our actions. This hypothesi s predicts a dissociation between moving and static objects in terms of the priming effects observed: Representations of static objects are long lasting and thus primed over temporal delays, whereas representations of moving objects are updated continuously and thus primed across image changes. 


\section{General methods}

Observers. Four Harvard undergraduate students participated in Experiment 1 and another four in Experiment 2. All observers had normal or corrected to normal vision and were naïve to the hypothesis under investigation.

Stimuli. In both experiments the stimuli consisted of 40 objects adapted from the asymmetrical characters of Tarr and Pinker (1990) as shown in Figure 2. The set of prime objects consisted of 10 asymmetrical figures The stimuli were presented on a 21 -inch colour monitor with a $1024 \times 768$ pixel resolution and $75 \mathrm{~Hz}$ refresh rate. The stimuli were drawn within a $4.82 \times 4.82$ degree of visual angle (DVA) square area on the screen.

Procedure and design. In both experiments the same immediate priming paradigm (Sekuler \& Palmer, 1992) was used as reported in previous studies (Kourtzi \& Shiffrar, 1997).

Figure 3 illustrates the experimental procedure. Each trial began with a fixation point presented for $1500 \mathrm{~ms}$, followed by the first prime frame shown for a variable duration to facilitate the perception of apparent motion. Then the second prime frame followed for the same duration as the first. A blank screen was then displayed for $500 \mathrm{~ms}$ followed by a pair of targets presented until the observer responded (with a $3 \mathrm{~s}$ maximum). The observers were instructed to carefully observe the prime objects and then press a key if the two subsequent targets matched each other. This "go-no go" task was used to reduce the variability often observed in priming studies that require observers to select one of two different motor responses (Biederman \& Gerhardstein, 1993). Observers were instructed that both reaction time and accuracy were important. Overall feedback (mean reaction time and percent correct responses) was provided at the end of each block of trials.
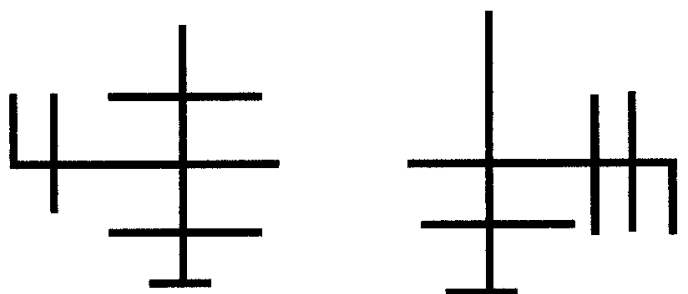

Figure 2. An example of the asymmetrical objects (Tarr \& Pinker, 1990) used as stimuli. The objects were similar configurations of line segments, with a 3.6 min line width, in different spatial arrangements. 


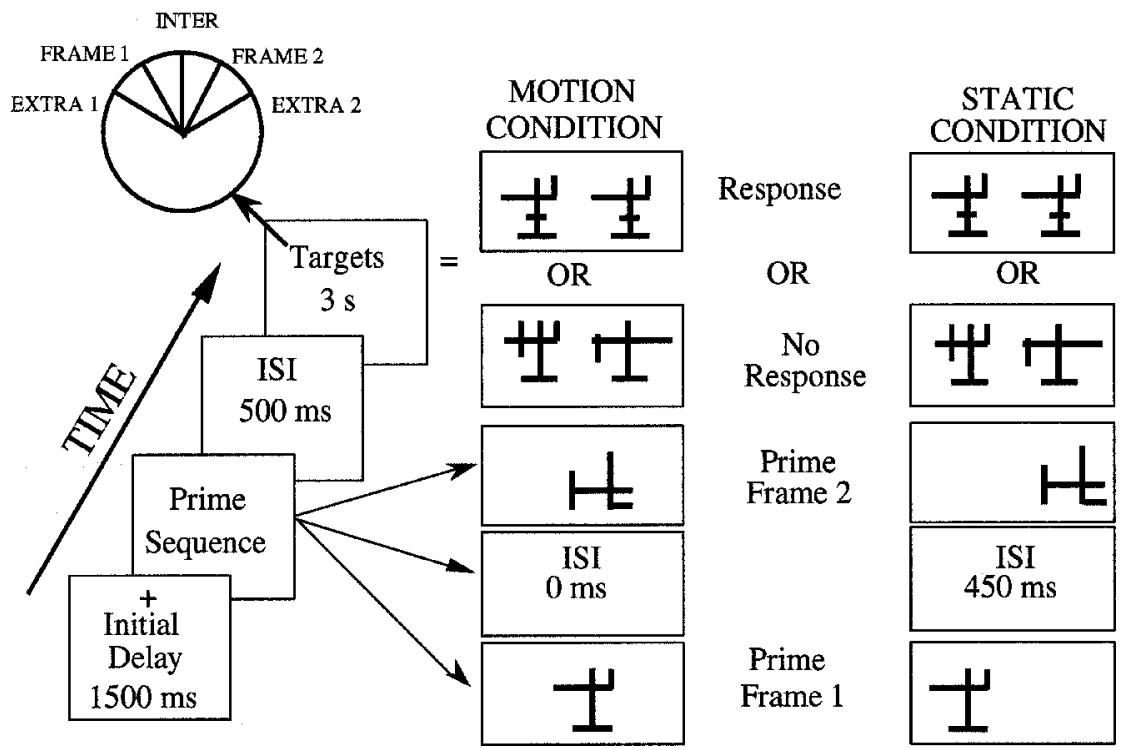

Figure 3. Experimental design for the apparent motion and static conditions.

The first prime had one of five possible orientations relative to the observer: $0^{\circ}$ (upright), $90^{\circ}, 180^{\circ},-45^{\circ},-135^{\circ}$. The second prime was a rotated version of the first in the frontal plane. The two primes were separated by rotation angles of $60^{\circ}$ or $150^{\circ}$. These angles were chosen based on previous studies (Kourtzi \& Shiffrar, 1997) showing different priming effects for small $\left(<90^{\circ}\right)$ and large rotation angles $\left(>90^{\circ}\right)$. The second prime was rotated clockwise from the first prime in half the trials and counterclockwise in the rest.

The duration of the two prime frames varied with the rotation angle between them such that optimal apparent motion was perceived. These durations were selected based on previous studies that used the same set of stimuli in this paradigm (Kourtzi \& Shiffrar, 1997). This yielded durations of 265 and $364 \mathrm{~ms}$ for the rotation angles of $60^{\circ}$ and $150^{\circ}$, respectively. The interstimulus interval (ISI) between the two prime frames was $0 \mathrm{~ms}$ in the apparent motion condition and $450 \mathrm{~ms}$ in the static condition. In the apparent motion condition, the first and second prime frames were presented so that the prime object appeared to rotate smoothly about its base. In the static condition, the second prime frame was displaced 2.41 DVA to the right of the first. This spatiotemporal separation between the two prime frames eliminated the perception of apparent motion in the static condition.

The targets were simultaneously presented 0.6 DVA to the left and right of the screen centre. The target objects were presented in one of five orientations: the first orientation of the prime (frame 1), the second orientation of the prime (frame 2), the orientation half way between the two prime orientations (inter), 
an orientation before the first prime orientation (extra 1), or an orientation beyond the second prime orientation (extra 2). The orientation of the inter target equalled the first orientation of the prime plus half the rotation angle. The extra 1 orientation equalled the first orientation of the prime minus half of the rotation angle. The extra 2 orientation equalled the second orientation of the prime plus half of the rotation angle. Thus, the orientation of the inter, extra 1 , and extra 2 targets all deviated equally from the prime orientations.

In both experiments, each condition (motion, static) was tested for each rotation angle $\left(60^{\circ}, 150^{\circ}\right)$ in separate blocks of trials. There were three types of trials: (1) trials in which the targets matched each other as well as the prime, (2) trials in which the targets matched each other but differed from the prime and (3) trials in which the targets differed from each other and the prime, (one-third of the total number of trials). Block order was counterbalanced across observers. Stimulus order was randomized within each block.

Data analysis. In all the experiments only reaction times to correct responses are reported because all observers exhibited ceiling levels of performance. The results are reported on the basis of observers and collapsed over items, first prime orientation and rotation direction (clockwise or counterclockwise) because no systematic pattern of differences was observed for these variables.

Priming is reported as the reaction time difference between trials in which the prime and targets were identical and trials in which the prime and targets differed. To guide the reader, we include in all the graphs from all the experiments the polar plots of the data from our previous studies that convey the characteristic signatures of the static and motion-based object representational systems (see Figure 1). This way, we can compare the priming data observed in each experiment to these characteristic signatures. This illustration of the data is useful for understanding how the representations of static and moving objects differ across the range of conditions tested in the three experiments reported.

Priming data are analysed to test for the signatures of the mechanisms for representation of moving and static objects. That is, we use ANOVAs with test frame (target orientations) and rotation angle $\left(60^{\circ}, 150^{\circ}\right)$ as independent variables to test for motion interpolation and suppression as well as static generalization in between and outside of the "known" views. Specifically, the motion interpolation effect is illustrated by non-significant differences in the amount of priming for the "known" views and the novel view falling within the motion path. The motion suppression effect is illustrated by significantly more priming for the "known" views than the novel views falling outside the motion path. The static generalization effect is illustrated by non-significant differences in the amount of priming for the "known" views and the novel views in between and outside the "known" views. 


\section{EXPERIMENT 1}

This experiment tested the hypothesis that representations of a moving target must be updated continuously across spatial changes so that we perceive the same object moving rather than multiple images of the object with different image properties. To this end, we tested how image changes, such as mirror reversals, size, or polarity changes affect the priming patterns that define the signatures of the motion-based and static object representational mechanisms. The observers were presented with two frame prime sequences either statically or in apparent motion and were tested with "known" and novel target views that had either the same properties as the prime views, were mirror reversed, presented in a different size, or had a different polarity from the prime views. Priming across these image changes for novel views within the objects' motion path but not between static "known" views would suggest that representations of moving objects are updated across spatial changes and thus are less spatially refined than the representations of static objects.

\section{Methods}

The observers completed four experimental sessions each consisting of six 75trial blocks (i.e., three blocks in the motion and three blocks in the static condition). In one session the targets were presented with the same properties as the primes. In each of the other three sessions the targets were presented mirror reversed from the primes, or in a different size (i.e., for half of the trials the targets were $33 \%$ larger from the primes and for the rest of the trials they were $33 \%$ smaller than the primes) or in a different polarity (i.e., the targets were white in grey background while the primes were always black in grey background). In each session for each condition and rotation angle there were 10 trials per target orientation in which the targets matched each other as well as the prime, and 10 trials in which the targets matched each other but differed from the prime.

\section{Results and discussion}

Figure 4 illustrates how the priming results observed across image changes compare to the characteristic signatures of the mechanisms for representing moving and static objects. Specifically, the results for static objects showed that the generalization mechanism fails across image changes. As shown in Figure 4 the priming data fit the elliptical plots only when there were no image changes applied to the test objects. Consistently, statistical tests on test frame (target orientations) for targets with the same image properties as the primes showed that the amount of priming for "known" views was not significantly different from the amount of priming for novel views in between the "known" views, $F(1,38)<1$. However, priming for "known" views was significantly lower than priming for novel views outside the "known" views, $F(1,38)=13.7$, 
I. NO IMAGE CHANGES

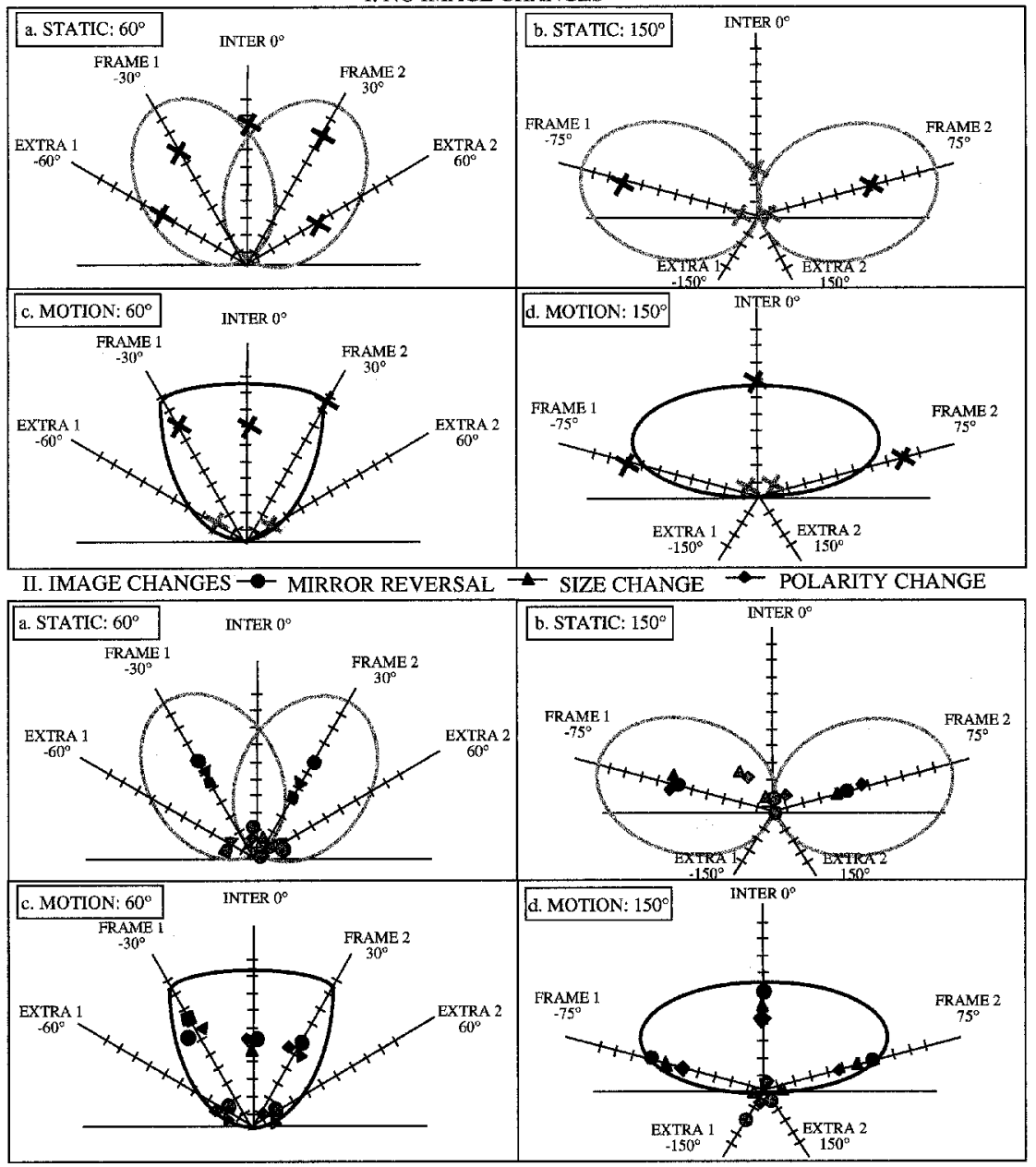

Figure 4. Results from Experiment 1. (I) Magnitude of priming for target orientations when targets were presented with the same properties as the primes in the static condition for rotation angles of (a) $60^{\circ}$ and (b) $150^{\circ}$, and in the apparent motion condition for rotation angles of (c) $60^{\circ}$ and (d) $150^{\circ}$. (II) Magnitude of priming for target orientations with image changes (i.e., mirror reversal, size change, polarity change ) in the static condition for rotation angles of (a) $60^{\circ}$ and (b) $150^{\circ}$, and in the apparent motion condition for rotation angles of (c) $60^{\circ}$ and (d) $150^{\circ}$. In all the data figures, the radial plots illustrate the priming magnitude in msec starting with $0 \mathrm{~ms}$ at the origin of the axes and increasing by steps of $10 \mathrm{~ms}$ towards the periphery of the radial plots. The data points in solid black illustrate significant priming effects while the data points in grey indicate non-significant priming. Data points plotted off the axes are non-significant negative priming values (i.e., reaction times were faster when the targets were different objects from the prime than the same as the prime) that fall on the extension of the axes representing orientations outside the "known" views. 
$p<.001$. Interactions between test frame (target orientations) and rotation angle $\left(60^{\circ}, 150^{\circ}\right)$ for novel views in between, $F(1,38)=2.3, p=.05$, and outside, $F(1,38)=5.1, p<.05$, "known" prime views indicates generalization for small but not for large rotation angles. However, significantly less priming was observed for novel views in between the "known" views when the targets were mirror reversed, $F(1,38)=5.5, p<.05$, or presented in a different size, $F(1,38)$ $=12.7, p=.001$, or different polarity, $F(1,38)=4, p=.05$, from the primes. Significantly less priming was also observed for novel views outside the "known" views when the targets were mirror reversed, $F(1,38)=9.7, p<.01$, or presented in a different size, $F(1,38)=12.9, p<.001$, or different polarity, $F(1,38)$ $=9.2, p<.01$, from the primes.

The results for moving objects showed an interpolation effect within the object's motion path and a suppression effect outside the motion path independent of rotation angle both when image changes were or were not applied to the targets. As shown in Figure 4 the priming data fit the curves describing the mechanism for representing moving objects. Consistently, statistical tests on test frame (target orientations) showed no significant differences in the priming between "known" views and novel views within the objects' motion path for targets with the same image properties as the primes, $F(1,38)<1$, mirror reversed targets, $F(1,38)<1$, targets that differed in size, $F(1,38)<1$, or targets that differed in polarity, $F(1,38)<1$. However, significantly less priming was observed for novel views outside the object's motion path than for "known" prime views for targets with the same image properties as the primes, $F(1,38)=$ $31.6, p<.001$, mirror reversed targets, $F(1,38)=12.2, p=.001$, targets that differed in size, $F(1,38)=22.8, p=.001$, or in polarity, $F(1,38)=17.1, p<.001$. These results replicate the characteristic signature of a mechanism for the representation of moving objects even across image changes.

Taken together, these results suggest that only the characteristic signature for the motion-based but not for the static object representational mechanism is replicated across image changes. In particular, the static generalization mechanism facilitates representation of objects within the space of similar views but fails when image changes increase the dissimilarity between novel and "known" views. Interestingly, in the static condition and for rotation angle of $60^{\circ}$ the amount of priming was higher for targets with the same properties as the prime than for targets mirror reversed or in different size or polarity from the prime. These results provide further evidence that image changes in the target presentation affect the static generalization mechanism. However, the motionbased mechanism appears capable of updating information across image changes that may occur as the objects move and facilitates the representation of objects across a range of different spatial configurations.

In sum, the current results suggest that the generalization gradient for moving objects and static views is very different; i.e., generalization across image changes (e.g., mirror reversal, size change, polarity change) is more extensive 
for moving than for static objects. Thus, these results provide further evidence that the representational mechanisms for static and moving objects are different. The static object mechanism is more spatially refined than the motionbased one. This fine spatial tuning is important for discriminating between similar objects from the same category, whereas rather broader spatial tuning may be necessary for the representation of moving objects that undergo various and continuous changes in their spatial configuration as they move.

\section{EXPERIMENT 2}

Experiment 1 provided evidence for a motion-based object mechanism that updates spatial information as objects move over time. Are these representations of moving objects stored permanently in memory or are they registered rather temporarily and updated continuously during the processing of the objects' motion?

To address this question we tested the memory characteristics of the motionbased and static object representational mechanisms. To this end, the observers were presented with prime sequences either statically or linked by apparent motion and tested with "known" and novel views one trial after the presentation of the prime sequences. We predicted that temporal delays would affect the memory of moving but not of static objects based on the hypothesis that the motion-based object representational mechanism updates information continuously to support visual guidance of our actions, whereas the static object mechanism supports rather long-lasting representations to serve processes of object recognition.

\section{Methods}

The stimuli, and the procedures were the same as in the motion and static conditions without any image changes on the targets used in Experiment 1 . The only difference was that the target views for each stimulus were tested one trial later after the presentation of the prime views. This temporal interval between a prime object and its corresponding targets depended on the duration of the primes on the current trial and the subjects' reaction time one trial back. For rotation angles of $60^{\circ}$ the total time to the presentation of the targets (i.e., sum of the initial delay, the prime presentation and the ISI between prime and targets) was $2530 \mathrm{~ms}$ for moving primes and $2980 \mathrm{~ms}$ for static primes. For rotation angles of $150^{\circ}$ the total time to the presentation of the targets was $2728 \mathrm{~ms}$ for moving primes and $3178 \mathrm{~ms}$ for static primes. The average reaction times of the subjects were lower than $1000 \mathrm{~ms}$. Thus, the temporal interval between a prime object and its corresponding targets was in the range of 3-4 s. 


\section{Results and discussion}

Figure 5 illustrates how the priming effects over temporal delays compare to the signatures of the representational mechanisms for moving and static objects. Specifically, the priming data for static objects fit the elliptical plots supporting restricted generalization effects. However, no priming was observed for moving objects either at known or novel orientations.

In the static condition statistical tests on test frame (target orientations) showed significantly stronger priming for "known" views than for novel views in between, $F(1,18)=3.1, p<.05$, or outside the "known" views, $F(1,18)=$ $10.5, p<.01$. An interaction, $F(1,18)=4.4, p=.05$, between test frame (target orientations) and rotation angle $\left(60^{\circ}, 150^{\circ}\right)$ supports a generalization effect for small but not for large rotation angles. In the motion condition, decreased priming for "known" and novel views was observed as well as no differences in the priming for "known" and novel views within or outside the objects' motion path, $F(1,18)<1$.

These results show differences in the time course of priming for moving and static objects. That is, priming for moving objects is more vulnerable to the effects of temporal delays, such that priming does not survive the presentation of an intermediate trial, whereas the priming for static objects is more robust under the same conditions. It is possible that these results are not due only to the long time interval between the prime and the targets but also to the interference, or memory load from the stimuli presented during the intermediate trial. It is important to note that the lack of priming for moving objects over temporal

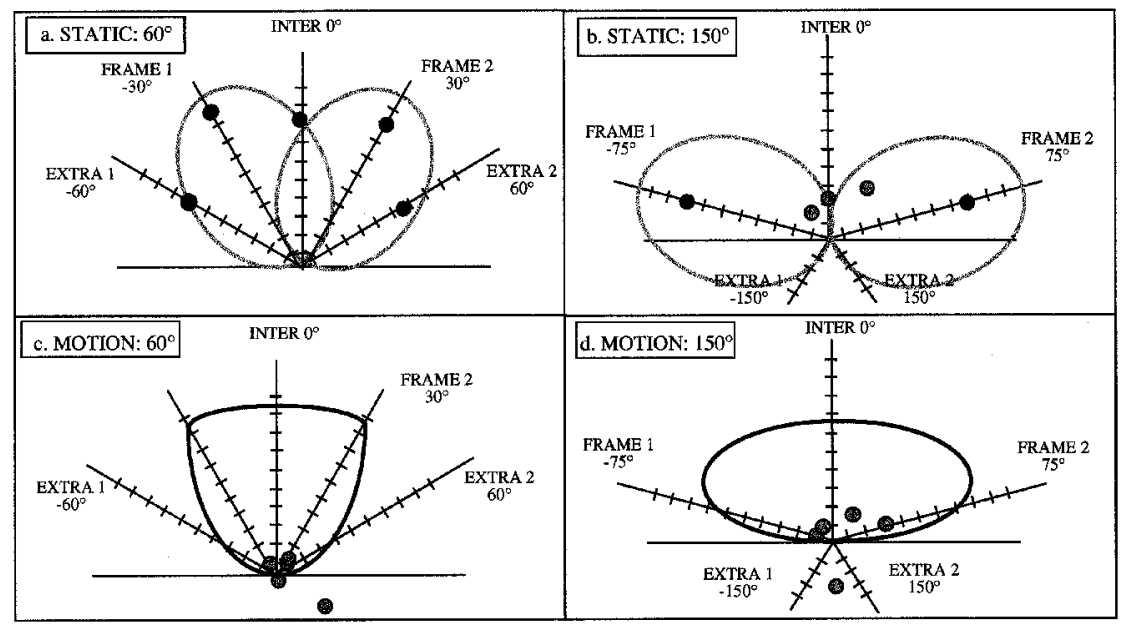

Figure 5. Results from Experiment 2. Magnitude of priming for target orientations tested after one intervening trial from the presentation of the prime sequences. Results in the static condition for rotation angles of (a) $60^{\circ}$ and (b) $150^{\circ}$, and in the motion condition for rotation angles of (c) $60^{\circ}$ and (d) $150^{\circ}$. 
delays was observed not only for novel but also for "known" views. That is, a "known" object view has a more temporary representation when presented as part of a motion sequence than as a static frame. This is yet another piece of evidence that there exist distinct representational mechanisms for moving and static objects. The visual system appears capable of integrating multiple images of a moving object to one entity and of updating its representation continuously. Long-lasting representations may be important for long-term recognition of static objects, whereas rather temporary representations may be more suitable for moving objects that change continuously as they move.

\section{GENERAL DISCUSSION}

The current experiments provide evidence for a representational mechanism for moving objects distinct from that for static objects. In particular, static objects are represented based on a generalization mechanism across object views within a limited spatial range. However, moving objects are represented based on an interpolation process between object views within the object's motion path even for long motion trajectories, but a suppression process outside this path. These effects define characteristic signatures for two distinct representational mechanisms: a static object mechanism based on the integration of multiple static images from an object, and a motion-based object mechanism tuned to a spatiotemporal sequence of images specific to a moving object.

These findings support an alternative approach to current theories of object recognition that was originally proposed by Freyd $(1987,1993)$ based on the representational momentum studies. That is, representations of objects are rather dynamic than fixed feature templates and may encode continuously updated information about objects during the analysis of their motion over time. An object's motion is not represented simply as a sequence of static frames linked by spatiotemporal associations (Lawson, Humphreys, \& Watson, 1994; Stone, 1998; Wallis, 1998). It seems that motion affects the way objects are represented by updating continuously information about objects during the analysis of their motion. Thus, dynamic representations of objects encode both space and time and may mediate the perception of events and actions (Freyd, 1993).

Moreover, the current results demonstrate that the static and motion-based mechanisms mediate object representations that have different characteristics. Specifically, static generalization lasts over temporal delays but fails across image changes. That is, this static object mechanism supports rather permanent but spatially refined representations. However, the motion-based mechanism updates continuously information about objects moving across space and time. As a result, representations of moving objects are less spatially refined and rather temporary compared to the representations of static objects. 
We propose that these distinct mechanisms for the representation of static and moving objects may mediate different cognitive processes. Specifically, the static object mechanism may mediate longer-term recognition and categorization of objects. However, the motion-based mechanism may support encoding of object properties in a "moment-to-moment" fashion (Ballard, Hayhoe, Pook, \& Rao, 1997; O'Regan, 1992) so that we can guide our actions towards moving objects as they change in space and time (Goodale \& Milner, 1992). Interestingly, object representations mediating actions have been shown to maintain information about size and orientation of objects in a rather temporary fashion (Goodale, Jackobson, \& Keillor, 1994; Goodale et al., 1991). In particular patient DF who suffers from visual form agnosia grasps objects at different sizes and orientations successfully when the objects are present but not when the objects have been viewed $2 \mathrm{~s}$ before. These studies support the hypothesis that the representational system for moving objects proposed by our current studies to mediate temporary representations of objects and to update them continuously across image changes may be involved in the visual guidance and perception of actions.

It is likely that these different cognitive representational processes for static and moving objects engage separate but interacting neural mechanisms that mediate perception of objects and guidance of actions directed at these objects (Goodale \& Humphrey, 1998). That is, the static object mechanism may involve occipito-temporal regions (Kourtzi \& Kanwisher, 2000b; Logothetis \& Sheinberg, 1996; Malach et al., 1995; Tanaka, 1996) responsible for shape processing, whereas the motion-based object mechanism may involve parietal regions engaged in the updating of the coordinates of visual targets for the visual guidance of movements towards them (Colby, 1998; Duhamel, Colby, \& Goldberg, 1992). Further neurophysiological and human imaging studies are required to investigate the neural substrates of these distinct mechanisms for the representation of static and moving objects.

\section{REFERENCES}

Ballard, D.H., Hayhoe, M.M., Pook, P.K., \& Rao, R.P. (1997). Diectic codes for the embodiment of cognition. Behavioral Brain Science, 20, 723-42.

Biederman, I., \& Gerhardstein, P.C. (1993). Recognizing depth-rotate d objects: Evidence and condition s for three-dimensiona 1 viewpoint invariance. Journal of Experimental Psychology: Human Perception and Performance, 19, 1162-1182.

Bülthoff, H.H., \& Edelman, S. (1992). Psychophysica 1 support for a 2-D view interpolation theory of object recognition. Proceedings of the National Academy of Science, 89, 60-64.

Colby, C.L. (1998). Action-oriented spatial reference frames in cortex. Neuron, 20, 15-24.

Cooper, E.E., Biederman, I., \& Hummel, J.E. (1992). Metric invarianc e in object recognition : A review and further evidence. Canadian Journal of Psychology, 46, 191-214.

Cooper, L.A., \& Schacter, D.L. (1992). Dissociation s between structural and episodic representations of visual objects. Current Directions in Psychological Science, 1, 141-146. 
Cooper, L.A., Schacter, D.L., Ballesteros, S., \& Moore, C. (1992). Priming and recognition of transformed three-dimensiona lobjects: Effects of size and reflection . Journal of Experimental Psychology: Learning, Memory, and Cognition, 18, 43-57.

Duhamel, J.R., Colby, C.L., \& Goldberg, M.E. (1992). The updating of the representation of visual space in parietal cortex by intended eye movements. Science, 255, 90-92.

Freyd, J.J. (1987). Dynamic mental representations . Psychological Review, 94, 427-438.

Freyd, J.J. (1993). Five hunches about perceptual processes and dynamic representations . In D. Meyer \& S. Kornblum (Eds.), Attention and performance XIV: Synergies in experimental psychology, artificial intelligence, and cognitive neuroscience (pp. 99-119). Cambridge, MA: MIT Press.

Freyd, J.J., \& Johnson, J.Q. (1987). Probing the time course of representationa 1 momentum. Journal of Experimental Psychology: Learning, Memory, and Cognition, 13, 259-268.

Freyd, J.J., \& Pantzer, T.M. (1995). Static pattern s moving in the mind. In S.M. Smith, T.B. Ward, $\&$ R.A. Finke (Eds.), The creative cognition approach (pp. 181-204). Cambridge, MA: MIT Press.

Goodale M.A., \& Humphrey, G.K. (1998). The objects of action and perception . Cognition, 67, 181-207.

Goodale, M.A., Jackobson, L.S., \& Keillor, J.M. (1994). Differences in the visual control of pantomimed and natural grasping movements. Neuropsychologia, 32, 1159-1178.

Goodale, M.A., \& Milner, A.D. (1992). Separate visual pathways for perception and action. Trends in Neuroscience, 15, 20-25.

Goodale, M.A., Milner, A.D., Jackobson, L.S., \& Carey, D.P. (1991). A neurologica 1 dissociation between perceiving objects and grasping them. Nature, 349, 154-156.

Kourtzi, Z., \& Kanwisher, N. (2000a). Activation in human MT/MST for static images with implied motion. Journal of Cognitive Neuroscience, 12, 1-8.

Kourtzi Z., \& Kanwisher, N. (2000b). Cortical regions involved in perceiving object shape. Journal of Neuroscience, 20, 3310-3318.

Kourtzi, Z., \& Shiffrar, M. (1997). One-shot view-invariance in a moving world. Psychological Science, 8, 461-466.

Kourtzi, Z., \& Shiffrar, M. (1999a). Dynamic representation s of human body movement. Perception, 28, 49-62.

Kourtzi, Z., \& Shiffrar, M. (1999b). The representation of three-dimensional, rotating objects. Acta Psychologica, 102, 265-292.

Kourtzi, Z., \& Shiffrar, M. (2001). The visual representation of malleable and rigid objects that deform as they rotate. Journal of Experimental Psychology: Human Perception and Performance, 27, 335-355.

Lawson, R., Humphreys, G.W., \& Watson, D.G. (1994). Object recognition under sequential viewing conditions : Evidence for viewpoint-specifi c recognition procedures. Perception, 23, 595-614.

Logothetis, N., Pauls, J., Bülthoff, H.H., \& Poggio, T. (1994). View-dependen t object recognition by monkeys. Current Biology, 4, 401-414.

Logothetis N., Pauls, J., \& Poggio, T. (1995). Shape representation in the inferior temporal cortex of monkeys. Current Biology, 5, 552-563.

Logothetis, N.K., \& Sheinberg, D.L. (1996). Visual object recognition . Annual Review of Neuroscience, 19, 577-621.

Malach, R., Reppas, J.B., Benson, R.B., Kwong, K.K., Jiang, H., Kennedy, W.A., Ledden, P.J., Brady, T.J., Rosen, B.R., \& Tootell, R.B.H. (1995). Object-relate d activity revealed by functional magnetic resonance imaging in human occipital cortex. Proceeding $s$ of the National Academy of Science USA, 92, 8135-8138.

Martin, A., Wiggs, C.L., Ungerleider, L.G., \& Haxby, J.V. (1996). Neural correlate s of category specific knowledge. Nature, 379, 649-652. 
O'Regan, J.K. (1992). Solving the "real" mysteries of visual perception: The world as an outside memory. Canadian Journal of Psychology, 46, 461-488.

Poggio, T., \& Edelman, S. (1990). A network that learns to recognize three-dimensiona 1 objects . Nature, 343, 263-266.

Reed, C.L., \& Vinson, N.G. (1996). Conceptual effects on representational momentum. Journal of Experimental Psychology: Human Perception and Performance, 22, 839-850.

Sakata, H., Taira, M., Kusunoki, A., Murata, A., \& Tanaka, Y. (1997). The parietal association cortex in depth perception and visual control of hand action. Trends in Neuroscience, 20, 350357.

Sakata, H., Taira, M., Kusunoki, A., Murata, A., Tanaka, Y., \& Tsutsui, K. (1998). Neural coding of $3 \mathrm{D}$ features of objects for hand action in the parietal cortex of the monkey. Philosophical Transactions of the Royal Society of London, B353, 1363-1373.

Sekuler, A.B., \& Palmer, S.E. (1992). Perception of partly occluded objects: A microgenetic analysis. Journal of Experimental Psychology: General, 121, 95-111.

Senior, C., Barnes, J., Giampietro, V., Brammer, M.J., Bullmore, E.T., Simmons, A., \& David, A.S. (2000). The functional neuroanatom y of implicit motion perception or "representationa 1 momentum". Current Biology, 10, 16-22.

Senior, C., Ward, J., \& David, A.S. (this issue). Representational momentum and the brain: An investigation into the functional necessity of V5/MT. Visual Cognition, 9, 81-92.

Shepard, R.N. (1981). Psychophysical complementary. In M. Kubovy \& J. Pomerantz (Eds.), Perceptual organization (pp. 279-341). Hillsdale, NJ: Lawrence Erlbaum Associates Inc.

Shepard, R.N. (1984). Ecological constraint s on internal representation : Resonant kinematic s of perceiving, imagining, thinking, and dreaming. Psychological Review, 91, 417-447.

Shepard, R.N. (1988). The role of transformation in spatial cognition. In J. Stiles-Davis, M. Kritchevsky, \& U. Bellugi (Eds.), Spatial cognition: Brain bases and development (pp. 81110). Hillsdale, NJ: Lawrence Erlbaum Associates Inc.

Shepard, R.N., \& Cooper, L.A. (1982). Mental images and their transformations . Cambridge, MA: MIT Press.

Shiffrar, M., \& Freyd, J.J. (1990). Apparent motion of the human body. Psychological Science, 1, $257-264$.

Shiffrar, M., \& Freyd, J.J. (1993). Timing and apparent motion path choice with human body photographs. Psychological Science, 4, 379-384.

Shiffrar, M., \& Shepard, R.N. (1991). Comparison of cube rotations around axes inclined relative to the environment or to the cube. Journal of Experimental Psychology: Human Perception and Performance, 17, 4-54.

Stevens, J.A., Fonlupt, P., Shiffrar, M., \& Decety, J. (2000). New aspects of motion perception: Selective neural encoding of apparent human movements. NeuroReport, 11, 109-115.

Stone, J.V. (1998). Object recognition using spatiotemporal signatures. Vision Research, 38, 947-951.

Tanaka, K. (1996). Inferotemporal cortex and object vision. Annual Review of Neuroscience, 19, $109-139$.

Tarr, M.J., \& Pinker, S. (1990). When does human object recognition use a viewer-centere d reference frame? Psychological Science, 1, 253-256.

Vinson, N.G., \& Reed, C.L. (this issue). Sources of object-specific effects in representationa 1 momentum. Visual Cognition, 9, 41-65.

Wallis, G. (1998). Spatio-temporal influence s at the neural level of object recognition . Network, 9 , $265-278$. 